\section{Aurélie Fontana Pierre D. Delmas}

\section{ADRESSE}

A. Fontana, P.D. Delmas: Service de rhumatologie et pathologie osseuse, Pavillon $F$ Hôpital Édouard-Herriot, 1, place d'Arsonval, 69437 Lyon Cedex, France.

$\mathrm{m} / \mathrm{s} \mathrm{n}^{\circ} 12$, vol. 17, décembre 2001

\title{
L'ostéoporose : épidémiologie, clinique et approches thérapeutiques
}

L'ostéoporose est un problème majeur de santé publique. On observe chaque année en France 50000 à 150000 nouveaux cas de fractures vertébrales et 50000 fractures de l'extrémité supérieure du fémur entraînant une morbidité et une mortalité accrue par rapport à la population générale. La définition de l'ostéoporose a évolué au cours des années et elle est actuellement définie par une masse osseuse faible et une détérioration de la micro-architecture osseuse conduisant à une fragilité osseuse et une augmentation du risque de fractures. L'absorptiométrie osseuse, qui mesure la densité minérale osseuse, permet de faire le diagnostic d'ostéoporose et de définir des groupes à risque de fractures. La stratégie thérapeutique a également évolué avec l'apparition au cours des dernières années de molécules ayant démontré leur efficacité pour réduire de 30 à $50 \%$ le risque de survenue de fractures vertébrales et non vertébrales au cours d'essais thérapeutiques contrôlés de grande ampleur. 0 utre le traitement hormonal substitutif, qui reste un traitement préventif de choix chez les femmes à risque, les bisphosphonates (alendronate et risédronate) et les modulateurs sélectifs des œstrogènes (raloxifène), représentent des traitements efficaces de l'ostéoporose. Le calcium et la vitamine D sont un traitement d'appoint utile, et leur utilisation doit être large chez les vieillards institutionnalisés. D'autres traitements, en particulier des stimulateurs de la formation osseuse, sont en cours d'évaluation.

'ostéoporose est une maladie générale du squelette caractérisée par une masse osseuse faible et une détérioration de a microarchitecture osseuse conduisant à une fragilité osseuse et une augmentation du risque de fractures [1] (figure 1). Le tissu osseux se renouvelle continuellement et la masse osseuse est maintenue par l'équilibre entre la résorption osseuse assurée par les ostéoclastes et la formation osseuse assurée par les ostéoblastes (voir les articles de R. Baron, P. Marie, et G. Karsenty, p. 1260 , p. 1252 et p. 1270 de ce numéro). L'ostéoporose résulte d'un déséquilibre entre la résorption et la 


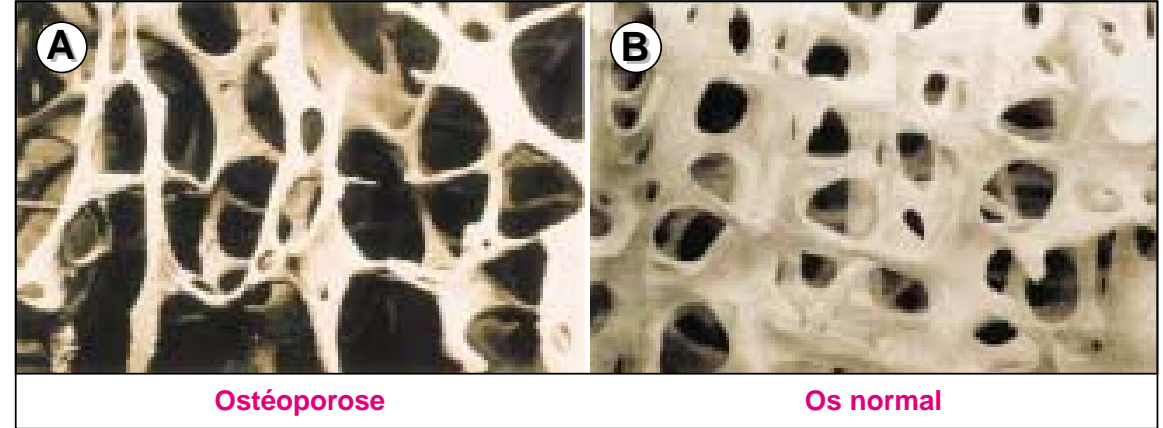

Figure 1. Diminution de l'épaisseur des travées osseuses et perte de leur interconnexion chez les patients ostéoporotiques.

formation osseuse. En fonction de l'étiologie de l'ostéoporose, l'augmentation de la résorption osseuse ou la diminution de la formation osseuse, comme c'est le cas dans l'ostéoporose cortisonique, seront prédominantes. Comme nous le verrons, de multiples facteurs interviennent dans l'ostéoporose et parmi eux, la carence œstrogénique joue un rôle fondamental, y compris chez I'homme. Elle entraîne une perte osseuse accélérée secondaire à l'augmentation de la résorption osseuse. Cette résorption accrue entraîne la perforation des travées osseuses et diminue la résistance mécanique du tissu osseux [2]. Le mécanisme d'action des œstrogènes est complexe car ils modulent aussi l'action d'hormones intervenant dans le métabolisme du calcium ( parathormone, vitamine $D$, calcitonine). Au niveau des cellules osseuses, les œstrogènes augmentent la sécrétion par les ostéoblastes de cytokines et facteurs de croissance qui inhibent la résorption osseuse. L'action directe des œestrogènes sur les ostéoclastes et la mise en évidence de récepteurs des œstrogènes sur les ostéoclastes restent encore discutées [3].

\section{Épidémiologie et impact socio-économique}

L'accroissement de l'espérance de vie fait émerger l'ostéoporose comme un problème majeur de santé publique. Parmi les femmes de 50 ans, $40 \%$ feront une ou plusieurs fractures par fragilité osseuse au cours de leur vie. Au-delà de 80 ans, $70 \%$ des femmes sont ostéoporotiques et $60 \%$ des femmes ostéoporotiques auront une médical de ces fractures ostéoporotiques est évalué entre 4 et 6 milliards de francs par an.

\section{Les fractures, principale complication de l'ostéoporose}

II survient chaque année en France 50000 fractures de l'extrémité supérieure du fémur dont 20 à $30 \%$ chez I'homme [5], mais l'incidence de ces fractures varie d'un pays à l'autre avec en particulier une incidence plus faible chez les noirs et les asiatiques [6]. Les fractures par ostéoporose sont grevées d'une morbidité et d'une mortalité importantes, surtout si elles touchent la hanche ou les vertèbres. Ainsi, une femme présentant une fracture de l'extrémité supérieure du fémur a un risque de décès dans I'année qui suit la fracture 2 à 4 fois supérieur à celui d'une femme du même âge dans la population générale [7]. La mortalité chez I'homme est plus élevée avec un taux de $10 \%$ à $14 \%$ dans le mois qui suit la fracture, contre $5 \%$ chez la femme [8]. La morbidité est également importante puisque 50 à $70 \%$ des patientes ayant eu une fracture de l'extrémité supérieure du fémur ne retrouvent pas leur autonomie antérieure [9].

L'incidence et la morbidité des fractures vertébrales sont mal documentées car il est difficile d'établir des critères diagnostiques précis et qu'il n'existe pas d'indice spécifique de la morbidité liée à la fracture vertébrale [10]. Chaque année on dénombre en France environ 50000 nouveaux cas de fractures vertébrales diagnostiquées, mais on estime à 150000 le nombre réél de fractures, car 2 fractures vertébrales sur 3 environ ne sont pas diagnostiquées. $L^{\prime}$ incidence chez l'homme est égale à la moitié de celle constatée chez les femmes [11]. Chez la femme, plus de $90 \%$ des fractures vertébrales surviennent à la suite d'un traumatisme minime, cette proportion étant de $55 \%$ chez I'homme [12].

La prédominance féminine des fractures du poignet est plus marquée ( $85 \%$ des fractures surviennent chez la femme) [13]. L'incidence chez la femme augmente de façon linéaire jusqu'à 60 ans puis reste stable audelà. Cette évolution peut s'expliquer en partie par le mécanisme des chutes qui, chez la personne âgée, se fait en général sans amortissement de la chute avec la main [14]. Ces fractures n'entraînent pas d'accroissement de la mortalité. D'autres fractures par ostéoporose touchent l'extrémité supérieure de l'humérus et du tibia, le bassin, les côtes. Ces fractures sont également plus fréquentes chez la femme et leur incidence augmente avec l'âge.

\section{Les conditions du diagnostic}

\section{Étiologie et facteurs de risque}

L'ostéoporose est le plus souvent une maladie multifactorielle. Si la carence œstrogénique secondaire à la ménopause joue un rôle déterminant dans la perte osseuse, de nombreux facteurs de risque s'y associent qui aggravent la perte osseuse et exposent au risque de fracture, notamment la corticothérapie. Les études épidémiologiques ont classé ces facteurs de risque en plusieurs catégories.

- Facteurs génétiques: le risque d'ostéoporose est accru chez un(e) patient(e) dont la mère a un antécédent de fracture vertébrale ou de I'extrémité supérieure du fémur. Des études conduites chez des jumeaux homozygotes ont démontré que la valeur du pic de masse osseuse avait une composante génétique importante chez les hommes et chez les femmes [15]. Plus la longueur du col du fémur est importante, plus cela favorise la survenue de fractures du col, et ce paramètre a également une composante génétique majeure [16]. 


\section{Étiologies et facteurs de risque fracturaire}

\section{Ménopause précoce \\ Génétique:}

fracture vertébrale ou de l'extrémité supérieure du fémur chez la mère

Facteurs nutritionnels et environnementaux :

apports calciques faibles

carence en vitamine D

consommation excessive d'alcool ou de tabac

vie sédentaire

maigreur

Événements fracturaires:

survenue d'un premier tassement vertébral

Pathologies: survenue d'une fracture non vertébrale

endocrinopathie

immobilisation prolongée

causes rares (mastocytose systémique, hépatopathie chronique,

Médicaments: entérocolopathies inflammatoires)

corticostéroïdes

hormones thyroïdiennes

héparine

analogues de la gonadotrophine releasing hormone

- Facteurs nutritionnels et environnementaux: un apport calcique faible (inférieur à $1 \mathrm{~g}$ par jour), associé ou non à une carence en vitamine $D$, ces deux facteurs étant fréquemment rencontrés chez des sujets âgés vivant en institution, favorisent la survenue d'une ostéoporose. Une faible exposition solaire, une consommation excessive de tabac ou d'alcool, une vie sédentaire, un faible rapport poids-taille sont autant de facteurs favorisant la perte osseuse [17].

- Les évén ements fracturaires: la survenue d'un premier tassement vertébral multiplie par 5 le risque que survienne un nouveau tassement, et ce indépendamment de l'âge et de l'importance de la masse osseuse. Une fracture touchant un os autre qu'une vertèbre augmente par 3 le risque de survenue d'un tassement vertébral $[18,19]$.

- Certaines pathologies e leur traitement: certaines maladies, la polyarthrite rhumatoïde, I'hyperthyroïdie, I'hyperparathyroïdie, les maladies inflammatoires de l'intestin, I'hypercorticisme, exposent au risque d'ostéoporose. La question se pose actuellement pour le SIDA et notamment chez les patients traités par des antirétroviraux, mais les données actuelles de la littérature ne permettent pas de conclure avec certitude.

\section{Données cliniques et biologiques}

L'interrogatoire recherche des antécédents familiaux d'ostéoporose, des antécédents personnels de fractures ou de douleurs rachidiennes aiguës, d'immobilisation prolongée ou d'hyperthyroïdie. II précisera l'âge de la ménopause, la consommation d'alcool et de tabac, la prise de corticoïdes ou de traitements à base d'hormones thyroïdiennes. L'interrogatoire évaluera aussi la ration calcique alimentaire. L'examen clinique recherche une perte de taille, des déformations du rachis, en particulier une cyphose dorsale, un contact entre les côtes et le bassin, tous ces éléments témmoignant de tassements vertébraux répétés.

Le bilan biologique sera d'autant plus approfondi que l'ostéoporose est sévère, notamment en cas de fractures vertébrales. Certains examens biologiques (vitesse de sédimentation, numération sanguine, calcémie, électrophorèse des protéines sériques, bilan thyroïdien) permettront d'éliminer certaines causes d'ostéoporose secondaire et certaines ostéopathies malignes.
$L$ 'augmentation du remodelage osseux, caractéristique de l'ostéoporose, peut être mise en évidence par le dosage de marqueurs de dégradation de la matrice osseuse ou par le dosage d'activités enzymatiques ou de protéines sécrétées par les cellules osseuses. On distingue les marqueurs de formation et de résorption osseuse [20].

Les marqueurs de la formation osseuse sont représentés par l'ostéocalcine, la phosphatase alcaline et les peptides d'extension du procollagène de type I (propeptides $\mathrm{N}$ et $\mathrm{C}$ ). L'ostéocalcine est une protéine non collagénique spécifique de l'os et de la dentine. Elle est synthétisée essentiellement par les ostéoblastes puis incorporée à la matrice extracellulaire. Une partie est libérée dans le sang circulant où elle peut être dosée. Les peptides d'extension du collagène sont clivés au moment de la maturation extracellulaire du collagène avant la formation des fibres. Ils reflètent également la formation osseuse car le collagène de type I est un composant abondant de la matrice osseuse. La phosphatase alcaline totale est le marqueur de formation osseuse le plus couramment utilisé, mais il manque de sensibilité. Des dosages reconnaissant l'isoenzyme osseux ont permis d'améliorer la spécificité et la sensibilité du dosage.

Les marqueurs de la résorption sont essentiellement représentés par la pyridinoline (Pyr) et la désoxypyridinoline (D-Pyr). Les autres marqueurs (calciurie, hydroxyprolinurie, phosphatase acide tartrate-résistante) manquent de spécificité et de sensibilité. La Pyr et D-Pyr sont des molécules de pontage entre les fibres de collagène de type I du tissu osseux, des tendons et de l'aorte (ces molécules sont absentes du collagène I de la peau). Au cours de la dégradation de la matrice osseuse, ces molécules sont libérées au sein des télopeptides du collagène de type I et peuvent être dosées à l'aide d'anticorps spécifiques dans le sang et l'urine.

Une estimation de l'intensité du remodelage osseux grâce à ces marqueurs présente un intérêt dans le suivi thérapeutique de l'ostéoporose où leur diminution à court terme au cours de certains traitements est prédictive de l'évolution à long terme de la densité minérale osseuse [21]. Les 
marqueurs sont aussi utiles au moment du diagnostic en améliorant l'évaluation du risque de perte osseuse ultérieure; des marqueurs augmentés indiquent que la vitesse de la perte osseuse sera importante [22].

\section{Mesure de la densité minérale osseuse}

Les techniques non invasives de mesure de la densité minérale osseuse permettent de faire le diagnostic d'ostéoporose. Plusieurs techniques de mesure existent. La technique des ultrasons a pour avantage d'utiliser des appareils de petite taille et peu coûteux. La valeur prédictive du risque fracturaire est bonne, mais la faible sensibilité aux variations $d u$ remodelage limite l'usage des ultrasons dans le suivi de l'ostéoporose [23]. La méthode de référence est l'absorptiométrie biphotonique aux rayons $X$ qui mesure la DMO (densité minérale osseuse) à différents sites: colonne lombaire, extrémité supérieure du fémur, avant-bras et corps entier. L'organisation mondiale de la santé a proposé une définition densitométrique de l'ostéoporose reposant sur la mesure du score T qui correspond à la différence en écart-type entre la valeur mesurée de la densité minérale osseuse du patient et la densité minérale osseuse maximale moyenne du jeune adulte de même sexe [24] (figure 2). De nombreuses études prospectives ont montré une corrélation entre la baisse de la DMO et I'augmentation du risque fracturaire [25-27]. Ainsi, l'ostéoporose n'est pas uniquement liée au vieillissement du tissu osseux et de nombreux facteurs interviennent, en particulier la carence œstrogénique chez la femme et des facteurs génétiques. Les moyens diagnostiques à notre disposition permettent de définir des personnes à risque de fractures et donc permettent d'intervenir avant la survenue de cette complication. Par ailleurs, les moyens thérapeutiques se sont considérablement développés depuis 5 ans permettant au clinicien d'envisager une prise en charge efficace de la maladie.

\section{Prise en charge thérapeutique}

\section{Les traitements}

Le T ableau I décrit les traitements de l'ostéoporose postménopausique disponibles en France. Le T ableau II rap- porte l'efficacité des traitements selon des critères d'évidence fondés sur les preuves (evidence based medecine).

\section{Le traitement hormonal substitutif (TH S)}

Le THS induit une augmentation de la densité minérale osseuse mesurée au niveau de la colonne vertébrale lombaire d'environ 5 à 10\% [28] Les données sur l'efficacité antifracturaire proviennent essentiellement d'études épidémiologiques d'observation. Le THS donné chez des patientes ménopausées réduit le risque relatif $(R R)$ de fracture vertébrale et du poignet $(R R=0,40)$ et de fracture de l'extrémité supérieure du fémur $(R R=0,60)$ [29]. Chez des patientes avec une ostéoporose avérée, le THS diminue également d'environ $50 \%$ le risque de survenue d'un tassement vertébral [30]. L'effet bénéfique du THS sur le risque fracturaire s'amenuise rapidement après arrêt du THS et n'est plus significatif 5 ans après l'arrêt du traitement. La perte osseuse postménopausique s'installant rapidement au cours des cinq années qui suivent la ménopause, le THS doit être instauré précocement, mais il garde un intérêt

Tableau I. Les traitements non œstrogéniques de l'ostéoporose postménopausique en France.

\begin{tabular}{|c|c|c|c|c|}
\hline Famille & Molécules & Posologie & Indications & Remboursement \\
\hline \multirow[t]{4}{*}{ Bisphosphonates } & $\begin{array}{l}\text { Etidronate } \\
\text { (Didronel }^{\circledR} \text { ) }\end{array}$ & $\begin{array}{l}400 \mathrm{mg} / \mathrm{j} 14 \mathrm{j} \\
\text { tous les } 3 \text { mois }\end{array}$ & $\begin{array}{l}\text { Ostéoporose } \\
\text { avec tassements }\end{array}$ & $\begin{array}{l}\text { Ostéoporose avec } \\
\text { tassements vertébraux }\end{array}$ \\
\hline & \multirow[t]{2}{*}{$\begin{array}{l}\text { Alendronate } \\
\left(\text { Fosamax }{ }^{\circledR}\right)\end{array}$} & $10 \mathrm{mg} / \mathrm{j}$ & $\begin{array}{l}\text { Ostéoporose avec } \\
\text { ou sans fracture }\end{array}$ & $\begin{array}{l}\text { Ostéoporose avec } \\
\text { fracture (tassement } \\
\text { vertébral, fracture } \\
\text { périphérique) }\end{array}$ \\
\hline & & $5 \mathrm{mg} / \mathrm{j}$ & $\begin{array}{l}\text { Femmes à risque } \\
\text { d'ostéoporose }\end{array}$ & Non remboursé \\
\hline & $\begin{array}{l}\text { Risédronate } \\
\text { (Actonel }{ }^{\circledR} \text { ) }\end{array}$ & $5 \mathrm{mg} / \mathrm{j}$ & $\begin{array}{l}\text { Ostéoporose avec } \\
\text { ou sans fracture et } \\
\text { traitement des femmes } \\
\text { à risque }\end{array}$ & $\begin{array}{l}\text { Ostéoporose avec } \\
\text { fracture (tassement } \\
\text { vertébral ou fracture } \\
\text { périphérique) }\end{array}$ \\
\hline SERM & Raloxifène & $60 \mathrm{mg} / \mathrm{j}$ & $\begin{array}{l}\text { Ostéoporose avec } \\
\text { traitement des femmes } \\
\text { à risque }\end{array}$ & $\begin{array}{l}\text { Ostéoporose avec } \\
\text { fracture (tassement } \\
\text { vertébral ou fracture } \\
\text { périphérique) }\end{array}$ \\
\hline \multirow{2}{*}{$\begin{array}{l}\text { Calcium et } \\
\text { vitamine D }\end{array}$} & Calcium & $1 \mathrm{~g} / \mathrm{j}$ & \multirow{2}{*}{$\begin{array}{l}\text { Insuffisances en } \\
\text { calcium et vitamine D } \\
\text { et traitement d'appoint } \\
\text { des ostéoporoses }\end{array}$} & \\
\hline & Vitamine D & 800 UI/j & & \\
\hline
\end{tabular}


Tableau II. Efficacité antifracturaire des traitements selon les critères d'évidence fondé sur les preuves. Analyse effectuée à partir d'études prospectives contrôlées, en double insu, dans lesquelles tous les malades reçoivent une supplémentation au calcium avec ou sans vitamine $D$.

\begin{tabular}{|c|c|c|}
\hline Traitement & racture vertébrale & Fracture non vertébrale \\
\hline œstrogènes ${ }^{1}$ & + & 0 \\
\hline alendronate & $+1+$ & + \\
\hline risédronate & $+1+$ & + \\
\hline étidronate & + & 0 \\
\hline raloxifène & H+ & 0 \\
\hline PTH 1-34 & $+1+$ & + \\
\hline calcitonine nasale & + & 0 \\
\hline sels de fluor & \pm & - \\
\hline analogues de la vitamine [ & \pm & 0 \\
\hline
\end{tabular}

${ }^{1}$ évidence fondée essentiellement sur des études d'observation.

++ : haut niveau de preuve; + : bon niveau de preuve; + : bas niveau de preuve; \pm : douteux; 0 : pas de preuve; - : augmentation du risque.

même à distance de la ménopause (vers 65 ans) [31]. Différentes modalités d'administration sont possibles avec un traitement combinant œstrogènes et progestatifs en continu (ce qui évite les hémorragies de privation) ou un traitement discontinu. II est instauré après la ménopause et doit être poursuivi longtemps ( 7 à 10 ans) pour permettre d'obtenir une réduction significative des fractures qui surviennent surtout après 65 ans. Les principaux effets secondaires induits par le THS sont les tensions mammaires, les œdèmes, les saignements irréguliers. L'augmentation du risque de cancer du sein sous THS a longtemps été source de controverse, et des études récentes ont montré une faible élévation du risque de cancer du sein pour des traitements prolongés au-delà de 10 ans [ 32].

\section{Les bisphosphonates}

L'ostéoporose est caractérisée par une augmentation du remodelage osseux avec un déséquilibre entre la résorption et la formation osseuse qui induit une perte osseuse accélérée. Les bisphophonates inhibent la résorption osseuse et ralentissent ainsi l'augmentation du remodelage osseux. Ils ont pour cible les ostéoclastes dont ils inhibent le recrutement et la différenciation, et dont ils modifient la fonction: ils diminuent son adhérence à la matrice osseuse, en diminuant la sécrètion d'enzymes nécessaires à la dissolution de la matrice osseuse [33] et ils augmentent I'apoptose des ostéoclastes [34]. En France, trois produits ont obtenu l'autorisation de mise sur le marché $\mathrm{m} / \mathrm{s} \mathrm{n}^{\circ} 12$, vol. 17, décembre 2001 de l'extrémite supérieure du fémur et du poignet [37]. Chez des patientes sans tassement vertébral mais avec une diminution de la DMO (score $T<-2$ ), la prise quotidienne de $10 \mathrm{mg}$ d'alendronate a aussi montré une réduction significative des fractures [38, 39]. L'alendronate entraîne une augmentation de la DMO qui atteint $8 \%$ à 3 ans au niveau de la colonne lombaire et une réduction des marqueurs du remodelage osseux qui atteignent en quelques mois les valeurs des femmes non ménopausées. L'alendronate donné à la dose quotidiennne de $5 \mathrm{mg}$ a montré son efficacité dans la prévention de l'ostéoporose chez des patientes récemment ménopausées. Au bout de 2 ans de traitement, on observe une augmentation de la DMO d'environ $3,5 \%$ à la colonne et $2 \%$ à la hanche alors que la DMO diminue à tous les sites sous placebo [40]. L'alendronate est bien toléré mais peut induire des symptômes gastriques et/ ou œsophagiens à type de brûlures.

En France, l'alendronate possède I'AMM dans le traitement de l'ostéoporose à la dose de $10 \mathrm{mg}$ par jour, mais le traitement n'est pris en charge par la sécurité sociale que dans les cas d'ostéoporose compliquée de fractures.

\section{- L e risédronate (A cton $\left.{ }^{\circledR}{ }^{\circledR}\right)$}

Deux études récentes, une nord-américaine et l'autre européenne, rapportent les effets antifracturaires du risédronate. La première étude inclut plus de 2400 femmes ayant au moins un tassement vertébral traité soit par placebo soit par risédronate $(5 \mathrm{mg} / \mathrm{j})$. Après 3 ans de traitement, les femmes traitées par risedronate ont une réduction significative de $40 \%$ du risque de survenue d'une nouvelle fracture vertébrale et ceci dès la première année. Le risque de fractures non vertébrales est également significativement diminué de $40 \%$ au bout de 3 ans de traitement dans le groupe risedronate comparé au groupe ayant reçu un placebo [41]. L'augmentation de la densité minérale osseuse est significativement plus élevée dans le groupe traité par risédronate $5 \mathrm{mg}$ comparé au placebo atteignant 5,4\% à la colonne lombaire, $1,6 \%$ au col fémoral et 3,3\% au trochanter. L'étude européenne et australienne 


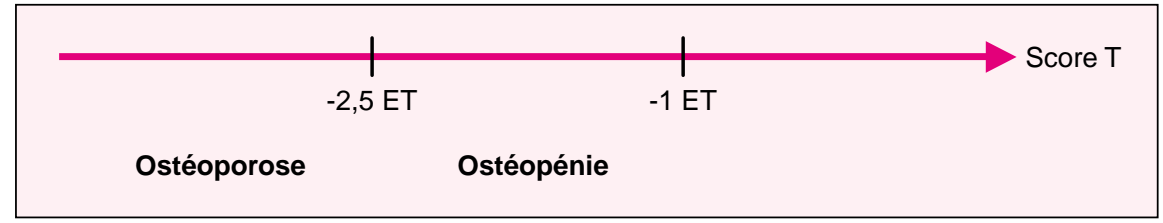

Figure 2. Définition de l'ostéoporose selon l'OMS. Le score T est la différence en écart type (ET) entre la valeur mesurée de la densité minérale osseuse du patient et la densité minérale osseuse maximale moyenne du jeune adulte de même sexe.

[42] a inclus 1226 patientes présentant une ostéoporose plus sévère avec au moins deux tassements vertébraux. Les femmes recevaient quotidiennement soit $5 \mathrm{mg}$ de risédronate, soit $2,5 \mathrm{mg}$ soit un placebo. La dose de $2,5 \mathrm{mg}$ a été arrêtée au bout de 2 ans, car les données intermédiaires ont montré un effet supérieur sur la densité minérale osseuse de la dose de $5 \mathrm{mg}$ avec un même profil de tolérance. Les résultats sont comparables à l'étude américaine avec une réduction d'environ $50 \%$ du risque de nouvelles fractures vertébrales et d'environ $30 \%$ du risque de fractures non vertébrales. L'effet du risédronate sur les fractures de hanche a été étudié chez près de 5500 femmes âgées de 70 à 80 ans avec une ostéoporose et près de 3900 femmes âgées de plus de 80 ans avec soit une DMO basse, soit au moins un facteur de risque de fracture de hanche. Les deux groupes recevaient 2,5 ou $5 \mathrm{mg}$ de risédronate par jour ou un placebo pendant 3 ans. L'analyse globale montre une diminution significative des fractures de hanche de $30 \%$ dans le groupe traité par risédronate. $L$ 'analyse des patients de 70 à 79 ans montre une diminution significative de $40 \%$ des fractures de la hanche dans le groupe traité par risédronate alors qu'il n'y a pas de différence significative chez les patientes de plus de 80 ans entre le groupe traité et le groupe placebo [43].

Le risédronate possède I'AMM dans la prévention et le traitement de I'ostéoporose postménopausique ainsi que dans la prévention de l'ostéoporose cortisonique chez les patientes ménopausées. Seule l'indication dans l'ostéoporose fracturaire bénéficie du remboursement.

Les SERM (selective estrogen receptor modulator)

Les analogues sélectifs des œstro- le tissu sur lequel elles agissent, se comportent comme des agonistes ou des antagonistes des œstrogènes. Le concept des SERM est né de la constation faite avec le tamoxifène, utilisé dans le cancer du sein pour son effet antiœstrogénique. Au niveau osseux, le tamoxifène se comporte comme un œstrogène et induit une augmentation de la DMO chez des patientes ménopausées traitées pour un cancer du sein [44]. Malheureusement, I'utilisation du tamoxifène chez des femmes ménopausées saines est difficile car le tamoxifène, s'il est utilisé de façon prolongée, induit une prolifération de la muqueuse endométriale avec un risque accru de cancer de l'endomètre.

\section{- Le raloxifène (Evista ${ }^{\circledR}$ )}

Dans une étude européenne multicentrique en double insu contre placebo, 600 femmes récemment ménopausées ont été traitées par raloxifène à la dose de 30,60 ou $150 \mathrm{mg} /$ jour pendant 2 ans $[45,46]$. Quelle que soit la dose, le raloxifène induit un gain minéral osseux modeste (de 2 à $4 \%$ ) mais significatif à la colonne lombaire, à la hanche et au corps entier contrastant avec la diminution significative sous placebo malgré l'adjonction de calcium, $500 \mathrm{mg} /$ jour. Dans l'étude MORE réalisée chez plus de 7700 femmes ostéoporotiques avec ou sans tassements vertébraux, le raloxifène à la dose de 60 ou $120 \mathrm{mg}$ réduit de 30 à $50 \%$ le risque de nouvelles fractures vertébrales après 2 et 3 ans de traitement, sans effet significatif sur les fractures non vertébrales [47]. En outre, le raloxifène réduit le cholestérol sérique et sa fraction LDL (d'environ $10 \%$ ). Parmi les effets indésirables, une légère augmentation des bouffées de chaleur ( $n$ 'induisant pas plus d'arrêt de traitement), des crampes dans les membres inférieurs, des thromboses veineuses périphériques (avec une incidence comparable à celle notée sous THS) et surtout une réduction de l'ordre de $75 \%$ de la survenue de cancer du sein chez les femmes ostéoporotiques [48, 49]. Le raloxifène est disponible en France dans la prévention et le traitement de l'ostéoporose postménopausique à la dose quotidienne de $60 \mathrm{mg}$ et il est remboursé dans le traitement de l'ostéoporose postménopausique avec fractures. II est actuellement contre-indiqué chez les patientes ayant un cancer du sein évolutif. Une étude est en cours chez les patientes à haut risque de cancer du sein.

\section{Le calcium et la vitamine D}

L'indication essentielle de la supplémentation vitamino-calcique est la prévention de la perte osseuse chez les personnes âgées, en particulier celles vivant en institution. Une étude a montré une diminution d'environ $25 \%$ du risque de fracture de l'extrémité supérieure du fémur chez des femmes âgées institutionnalisées [50]. Chez les femmes ménopausées, l'apport alimentaire en calcium étant souvent insuffisant, on associe souvent au traitement antiostéoporotique un apport de 500 à $1000 \mathrm{mg} / \mathrm{j}$ de calcium et de 400 à $800 \mathrm{UI} / \mathrm{j}$ de vitamine $\mathrm{D}$.

\section{Autres produits}

Le fluor a longtemps été utilisé comme traitement de l'ostéoporose, mais aucune étude n'a démon tré son efficacité dans la prévention des fractures. L'étude FAVOS [51] n'ayant pas montré de supériorité du fluor sur l'association calcium-vitamine D dans la prévention des fractures vertébrales, on tend actuellement en France à ne plus utiliser le fluor dans le traitement de l'ostéoporose. De plus, la fluorothérapie pourrait augmenter le risque de fracture de l'extrémité supérieure du fémur. La calcitonine injectable est parfois utilisée en France pour ses propriétés antalgiques dans le traitement de la phase aiguë postfracturaire. Par ailleurs, une étude américaine [52] (PROOF) a montré une réduction modeste des fractures vertébrales chez des femmes ostéoporotiques traitées 5 ans par calcitonine nasale 
$200 \mathrm{UI} / \mathrm{j}$. Les métabolites actifs de la vitamine $D(1 \alpha O H$ vitamine $D$ et 1,25 dihydroxyvitamineD) sont autorisés dans le traitement de l'ostéoporose dans certains pays, sur la base d'études peu convaincantes.

\section{Perspectives}

Contrastant avec l'abondance des traitements antirésorptifs, il n'y a actuellement pas de traitement disponible capable de stimuler la formation osseuse (anabolisants osseux). Le ranelate de strontium, en cours de développement, pourrait inhiber la résorption osseuse et stimuler la formation osseuse et des études de phase III sont en cours. Chez I'animal, les statines agissent comme de puissants stimulateurs de la formation osseuse ostéoblastique mais les études épidémiologiques effectuées chez les utilisateurs de statines sont contradictoires [53].

Actuellement, de grands espoirs sont fondés sur l'utilisation d'un fragment de la parathormone humaine (le fragment contenant les acides aminés 1 à 34), injectée quotidiennement à la dose de $20 \mu \mathrm{g}$. Dans une étude très récente chez plus de 1600 femmes avec une ostéoporose vertébrale fracturaire, l'incidence des fractures vertébrales et non vertébrales était réduite de $65 \%$ et $55 \%$ respectivement à l'issue des 21 mois de traitement, avec des effets indésirables modérés et peu nombreux [54]. La possibilité d'utiliser la PTH de façon séquentielle avec les antirésorptifs permet d'envisager de nouvelles stratégies thérapeutiques.

\section{Indications thérapeutiques}

\section{En cas de fractures récentes (dans les} années qui précèdent)

En cas de fracture vertébrale, le traitement fera appel de préférence aux bisphosphonates de deuxième génération (alendronate, risedronate) ou au raloxifène. En cas de contre-indications ou d'effets secondaires à l'utiliation de ces traitements, l'étidronate peut être proposé. En cas de fractures non vertébrales (fractures périphériques, fractures de hanche) ou en cas de risque élevé de fracture de l'extrémité supérieure du col du fémur, on fera appel de préférence à I'alendronate ou au risedronate. Des études ont montré un effet positif sur la densité minérale osseuse de l'association alendronate-THS ou alendronate-raloxifène, mais le bénéfice supplémentaire de telles associations sur le risque fracturaire n'est pas connu.

\section{En l'absence de fractures récentes}

La décision thérapeutique repose la mesure de la densité minerale osseuse, sur l'âge et sur l'analyse des facteurs de risque de fractures.

- Si le T score est inférieur à - 2,5 (ostéoporose), les indications thérapeutiques sont théoriquement les mêmes que celles faites en cas de fractures récentes mais sont à moduler en fonction de l'âge, de l'importance de la perte osseuse et des facteurs de risque associés. Aucun des traitements n'est, à ce jour, pris en charge par la sécurité sociale dans cette indication en dehors du THS.

- Si le T score est compris entre - 2,5 et - 1 (ostéopénie), l'instauration d'un traitement préventif est à discuter au cas par cas. Un traitement antirésorptif, notament THS ou raloxifène, sera volontiers proposé si la densité osseuse est basse (score T entre - 2,5 et - 2) ou s'il existe d'autres facteurs de risque fracturaire. Chez les personnes de plus de 75 ans, on se contentera le plus souvent d'une supplémentation en calcium et vitamine $D$.

\section{Le suivi thérapeutique}

L'efficacité du traitement de l'ostéoporose est jugé sur l'absence de survenue de nouvelles fractures. Mais, l'effet des traitements n'est pas immédiat et la plupart des produits utilisés ont montré leur efficacité antifracturaire au bout d'une année. La survenue d'une fracture dans la première année de traitement ne doit donc pas être considérée comme un échec thérapeutique.

La mesure de la densitométrie osseuse est utilisée dans le suivi de I'ostéoporose. La plupart des études évaluant les traitements de l'ostéoporose montre une augmentation significative de la densité minérale osseuse dès la première année de traitement. En pratique clinique, la surveillance se fait tous les deux ans, car à l'échelle individuelle, du fait des variations de mesure imputables à la technique, les faibles variations observées à un an chez le patient, sont difficilement interprétables. L'ostéodensitométrie est prise en défaut dans le suivi des traitements par le raloxifène qui n'entraîne qu'une augmentation faible ( 2 à $3 \%$ ) de la densité minérale osseuse, du même ordre de grandeur que l'erreur de mesure imputable à la technique.

Plusieurs études ont montré que les marqueurs du remodelage osseux avaient un intérêt dans le suivi thérapeutique car leur diminution après 3 à 6 mois de traitement est prédictive de l'augmentation de la densité osseuse que les patients traités auront après deux ans de traitement [20].

\section{La prise en charge générale du malade ostéoporotique}

La prise en charge du patient ostéoporotique ne relève pas uniquement de la prescription médicamenteuse. II est admis que l'exercice physique a un effet bénéfique sur la masse osseuse bien que le type d'exercice et la quantité ne soient pas clairement définis. En revanche, chez les patients ostéoporotiques, une activité augmentant les risques de chute est déconseillée alors que la marche est particulièrement indiquée. Une activité physique modérée présente également I'avantage de renforcer la masse musculaire, aidant ainsi dans la prévention des chutes. En cas de fracture vertébrale récente ou de douleurs rachidiennes chroniques, la prise en charge de la douleur est également importante et parallèlement aux médications antalgiques, les corsets sur mesure de maintien lombaires renforcés peuvent apporter un bénéfice antalgique.

Les chutes constituent la première cause d'accident chez les personnes âgées et peuvent entraîner des fractures en cas d'ostéoporose. Différents conseils peuvent être donné pour limiter ces chutes:

- aménager I'habitat: éclairage de bonne qualité, rampes dans les sanitaires et les escaliers, éviter les tapis; - adapter la tenue vestimentaire: port de chaussures maintenant bien le pied, semelles antidérapantes, éviter les vêtements amples;

- veiller à dépister les déficits visuels, les troubles de l'équilibre. 


\section{Cas particulier de l'ostéoporose masculine}

L'ostéoporose ne concerne pas que les femmes; $30 \%$ des fractures de l'extrémité supérieure du fémur surviennent chez l'homme et l'incidence des fractures vertébrales est égale à environ la moitié de celle des femmes.

Différents facteurs peuvent expliquer une incidence plus faible de l'ostéoporose chez l'homme. Le capital osseux maximal acquis entre 20 et 30 ans est supérieur d'environ $30 \%$ à celui de la femme. La taille des os longs et des vertèbres est également plus élevée avec une résistance biomécanique accrue. La microarchitecture osseuse semble également mieux conservée [55]. Contrairement aux femmes chez qui la carence œstrogénique est majeure après la ménopause, la diminution des hormones mâles avec l'âge est progressive et moins marquée chez l'homme. Par ailleurs, les hommes ont une espérance de vie plus courte qui explique aussi la moindre incidence de l'ostéoporose par rapport à la femme.

Le diagnostic d'ostéoporose par absorptiométrie est également applicable chez l'homme et il est habituel d'utiliser comme seuil diagnostic un score $T$ à $-2,5$ identique à celui de la femme, mais ce seuil n'est pas encore définitivement établi.

Les traitements utilisés dans I'ostéoporose de la femme ne bénéficie pas de I'AMM dans l'ostéoporose primitive masculine mais des données récentes montrent une efficacité de l'alendronate chez I'homme [56]. Un groupe de 241 patients avec une ostéoporose primitive ont été traités pendant 2 ans contre placebo par $10 \mathrm{mg}$ d'alendronate. La densité minérale osseuse augmente significativement dans le groupe traité d'environ $7 \%$ à la colonne lombaire et $2,5 \%$ au col fémoral. Dans le groupe placebo, elle augmente significativement d'environ $2 \%$ au niveau de la colonne lombaire et reste stable au col fémoral. L'incidence des fractures vertébral es est significativement diminuée dans le groupe alendronate comparée au placebo ( $0,8 \%$ ver-

\section{Conclusions}

Le traitement de la perte osseuse postménopausique et de l'ostéoporose fait actuellement appel à des molécules qui inhibent la résorption osseuse. Alors que le THS est surtout utilisé dans la prévention de la perte osseuse, l'alendronate, le risédronate et le raloxifène ont démontré de façon non équivoque leur capacité à réduire de façon marquée l'incidence des fractures vertébrales et représentent le traitement de choix de I'ostéoporose vertébrale. Par ailleurs, les SERM représentent un traitement de choix dans la prévention de la perte osseuse postménopausique chez des patients ayant eu un cancer du sein, et leur inocuité est en cours d'évaluation chez les patientes à haut risque. La survenue prochaine de la PTH va renforcer notre arsenal thérapeutique, notamment dans les ostéoporoses les plus sévères

\section{RÉFÉRENCES}

1. Consensus development conference. Prophylaxis and treatment of osteoporosis. A m J M ed 1991; 90 : 107-10.

2. Pacifici R. Estrogen, cytokines, and pathogenesis of postmenopausal osteoporosis. J Bone M iner Res 1996; 11: 1043-51.

3. O ursler MJ, O sdoby P, Pyfferoen J, et al. Avian osteoclasts as estrogen target cells. Proc Natl Acad Sci USA 1991; 88: 6613-7.

4. Melton LJ. How many women have osteoporosis now? J Bone M iner Res 1995; 10: 175-7.

5. Cooper C, Campion G, Melton LJ. Hip fractures in the elderly : a world-wide projection. Osteoporos Int 1992; 2 : 285-9.

6. Melton LJ. Differing patterns of osteoporosis across the world. In: Chesnut $\mathrm{CH}$, ed. $\mathrm{New}$ dimensions in osteoporosis in the 1990s. Asia Pacific Conference Series $n^{\circ} 125$. Hong Kong: Excerpta Medica, 1991: 13-8.

7. Schurch MA, Rizzoli R, Mermillod B, et al. A prospective study on socioeconomic aspects on fracture of the proximal femur. J Bone M iner Res 1996; 11: 1935-42.

8. Kellie SE, Brody JA. Sex-specific and racespecific hip fractures rates. Am J Publ $\mathrm{H}$ ealth 1990; 80: 326-8.

9. Marotelli RA, Berkman LF, Cooney LM, et al. Decline in physical function following hip fracture. J Am Geriatr Soc 1992; 40: 861-6.
10. Kanis JA, McCloskey EV. Epidemiology of vertebral osteoporosis. Bone 1992; 13: S1-10.

11. Cooper C, Atkinson EJ, O'Fallen WM, et al. Incidence of clinically diagnosed vertebral fractures: a population-based study in Rochester, Minnesota, 1985-1989. J Bone $M$ iner Res 1992; $7: 221-7$.

12. Melton LJ, Lane AW, Cooper $C$, et al. Prevalence and incidence of vertebral deformities. Osteoporos Int 1993; 3: 113-9.

13. O wen RA, Melton LJ, Johnson KA, et al. Incidence of Colls' fractures in a north american community. Am J Publ Health 1982; 72: 605-7.

14. Cooper C, Melton LJ. Epidemiology of osteoporosis. Trends Endocrinol M etab 1992; 314 : 224-9.

15. Pocock NA. Genetic determinants of bone mass in adults. J Clin Invest 1987; 80 : 706-10.

16. Cummings SR, Cauley J, Palermo L, et al. Racial differences in hip axis lengths might explain racial differences in rates of hip fracture. Osteoporos Int 1994 ; 4 : 226-9.

17. Johnell O, Gullberg B, Kanis JA, et al. Risk factors for hip fracture in European women : the MEDOS study. J Bone M ine Res $1995 ; 10$ : 1802-15.

18. Ross PD, Davis JW, Epstein RS, Wasnish $\mathrm{RD}$. Pre-existing fractures and bone mass predict vertebral fracture incidence in women. Ann Intern M ed 1991; 114: 914-23.

19. Wasnich RD, Davis JW, Ross PD. Spine fracture risk is predicted by non-spine fractures. Osteoporos Int 1994; 4: 1-5.

20. Delmas PD, Eastell R, Garnero P, et al. The use of biochemical markers of bone turnover in osteoporosis. Osteoporos Int 2000; suppl 6: S2-17.

21. Garnero P, Shih WJ, Gineyts E, et al. Comparison of new biochemical markers of bone turnover in late postmenopausal osteoporotic women in response to alendronate treatment. J Clin Endocrinol M etab 1994; 79: 1693-700

22. Garnero P, Sornay-Rendu E, Duboeuf $F$, Delmas P. Biochemical markers of bone turnover predict postmenopausal forearm bone loss over 4 years: the OFELY study. J Bone M iner Res 1999; 14 : 1614-21.

23. Hans D, Dargent-Molina P, Schott AM, et al. UItrasonographic heel measurements to predict hip fracture in elderly women: the EPIDOS prospective study. Lancet 1996 ; 348: 511-4.

24. Kanis JA, Melton LJ, Christiansen $C$, et al. The diagnosis of osteoporosis. J Bone $M$ iner Res 1994; 9 : 1137-41.

25. Gardsell $P$, Johnell $O$, Nilsson BE. The predictive value of bone loss for fragility fractures in women: a longitudinal study over 15 years. Calcif Tissue Int 1991; 49: 90-4.

26. Black DM, Cummings SR, Genant HK, et al. Axial and appendicular bone density predict fractures in older women. J B one M iner Res 1992; $7: 633-8$. 


\section{RÉFÉRENCES}

27. Cummings SR, Black DM, Newitt MC, et al. Bone density at various sites for prediction of hip fractures. Lancet 1993; 341: 72-5.

28. Hesley PP, Shepard KA, Jenkins DK, et al. Monitoring estrogen replacement therapy and identifying rapid bone losers with an immunoassay for deoxypyridinoline. 0 steoporos Int 1998; 8 : 159-64.

29. Cauley JA, Seeley DJ, Ensrud K, et al. Estrogen replacement therapy and fractures in older women. Ann Intern M ed 1995; 122 : 9-16.

30. Lufkin EG, Wahner HW, O'Fallon WM, et al. Treatment of postmenopausal osteoporosis with transdermal estrogen. Ann intern M ed 1992; 117: 1-9.

31. Felson DT, Zhang Y, Nannan MT, et al. The effect of postmenopausal estrogen therapy on bone density in elderly women. $\mathrm{N}$ Engl J M ed 1993; 329: 114-6.

32. Collaborative Group on Hormonal therapy in breast cancer. Breast cancer and hormone replacement therapy: collaborative reanalysis of data from 51 epidemiological studies of 52705 women with breast cancer and 108411 women without breast cancer. Lancet 1997; 350: 1047-59.

33. Lin JH. Bisphosphonates: a review of their pharmacokinetic properties. Bone 1996; 18: 75-85.

34. Luckman SP, Hughes DE, Coxon FP, et al. Nitrogen-containing bisphosphonates inibit the mevalonate pathway and prevent post-translational prenylation of GTP-binding proteins, including Ras. J Bone Miner Res 1998; 13: 581-9.

35. Storm T, Thamsborg G, Steiniche $T$, Genant HK 'Sorensen OH. Effect of intermittent cyclical etidronate therapy on bone mass and fracture rate in women with postmenopausal osteoporosis. N Engl J M ed 1990; 322: 1265-71.

36. Watts NB, Harris ST, Genant HK et al. Intermittent cyclical etidronate treatment of postmenopausal osteoporosis. N Engl J $M$ ed 1990; 323: 73-9.

37. Black DM, Cummings SR, Karpf DB, et al. Randomized trial of effect of alendronate on risk fracture in women with existing vertebral fractures. L ancet 1996; 348: 153541.

38. Cummings SR, Black DM, Thompson $D E$, et al. Effect of alendronate on risk fracture in women with low bone density but without vertebral fracture. JAM A 1998; 280 : 2077-82.

39. Pols HAP, Felsenberg D, Hanley DA, et al. Multinational, placebo-controlled, randomised trial of the effects of alendronate on bone density and fracture risk in postmenopausal women with low bone mass: results of the FOSIT study. Osteoporos Int $1999 ; 9$ : 461-8

40. Hosking D, Chilvers CED, Christiansen $C$, et al. Prevention of bone loss with alen$\mathrm{m} / \mathrm{s} \mathrm{n}^{\circ} 12$, vol. 17, décembre 2001 dronate in postmenopausal women under 60 years of age. $N$ Engl] M ed 1998; 338: 48592.

41. Harris ST, Watts NB, Genant $\mathrm{HK}$ \& al. Effects of risedronate treatment vertebral and non vertebral fractures in women with postmenopausal osteoporosis. A randomised controlled trial. JAM A 1999; 282: 1344 52.

42. Reginster JY, Minne HW, Sorensen $\mathrm{OH}$, et al. Randomized trial of the effects of risedronate on vertebral fractures in women with established postmenopausal osteoporosis. Osteoporos Int 2000; 11:83-91.

43. McClung MR, Geusens P, Miller PD, et al. Effect of risedronate on the risk of hip racture in elderly women. Hip Intervention Program Study Group. N Engl J M ed 2001 ; 344 : 333-40.

44. Powles TJ, Hickish $T$, Kanis JA, et al. Effect of tamoxifen on bone mineral density measured by dual-energy X-ray absoptiometry in healthy premenopausal and postmenopausal women. J Clin Oncol 1996; $14: 78-84$.

45. Delmas PD, Bjarnason NH, Mitlak BH, et al. The effects of raloxifene on bone mineral density, serum cholesterol, and uterine endometrium in postmenopausal women. $\mathrm{N}$ Engl J M ed 1997; 337: 1641-7.

46. Johnston CC Jr, Bjarnason $\mathrm{NH}$, Cohen Fj, et al. Long-term effects of raloxifene on bone mineral density, bone turnover, and serum lipid levels in early postmenopausal women: three-year data from 2 doubleblind, randomized, placebo-controlled trials. Arch Intern M ed 2000; 160: 3444-50.

47. Ettinger $B, B l a c k D M$, Mitlak $B H$, et al. Reduction of vertebral fracture risk in postmenopausal women with osteoporosis treated with raloxifene. Results from a 3-year randomized clinical trial. JAM A 1999; 282: $637-45$.

48. Cummings SR, Eckert S, Krueger KA, et al. The effect of raloxifene on risk of breast cancer in postmenopausal women. JAM A $1999 ; 281$ : 2189-97.

49. Cauley JA, Norton L, Lippman ME, et al. Continued breast cancer risk reduction in postmenopausal women treated with raloxifene: 4-year results from the more trial. Multiples outcome of raloxifène evolution. Breast Cancer Res Treat 2001; 65: 125-34.

50. Chapuy MC, Arlot ME, Delmas PD, et al. Effect of calcium and cholecalciferol treatment for three years on hip fractures in elderly women. Br M ed J 1994; 308: 1081-2.

51. Meunier PJ, Sebert JL, Reginster JY, et al. Fluoride salt are no better at preventing new vertebral fractures than calcium-vitamin D in post-menopausal osteoporosis: the FAVOS study. Osteoporos Int 1998; 8: 4-12.

52. Chesnut $\mathrm{CH}$, Silverman $\mathrm{S}$, Andriano $\mathrm{K}$, et al. A randomized trial of nasal spray salmon calcitonin in postmenopausal women with established osteoporosis: the prevent recurrence of osteoporotic fractures study. PROOF study group. Am J M ed 2000; 109: 330-1.
53. van Staa TP, Wegman $S$, de Vries F, et al. Use of statins and risk of fractures. IAMA 2001 ; 285: 1850-5.

54. Neer RM, Arnaud CD, Zanchetta JR, et al. Effect of parathyroid hormone (1-34) on fractures and bone mineral density in postmenopausal women with osteoporosis. N Engl J M ed 2001; 344: 1434-41.

55. Mosekilde L. Sex differences in age-related changes in vertebral body size, density and biochemical competence in normal individuals. Bone 1990; 11: 67-73.

56. O rwoll $E$, Ettinger $M$, Weiss $S$, et al. Alendronate for the treatment of osteoporosis in men. N Engl J M ed 2000; 343: 604-10.

\section{Summary}

O steoporosis: epidemiology and treatment

O steoporosis is a systemic skeletal disease characterized by a low bone mineral density and microarchitectural deterioration of bone tissue, leading to an increase fracture risk. If untreated, almost half of white women 50 years of age will experience one or more osteoporotic fracture during their lifetime. Therefore, osteoporosis represents a major public health problem. Bone mineral density measurement allows to quantify accurately the risk of fragility fractures. Hormonal replacement therapy is the treatment of choice in the prevention of osteoporosis. Bisphosphonates (alendronate, risedronate) and the selective estrogen receptor modulator (raloxifene) have demonstrated, in large placebocontrolled studies, their efficacy to reduce the risk of osteoporotic fracture by about $30 \%$ to $50 \%$. Among stimulators of bone formation, parathyroid hormone has been shown recently to also reduce the risk of vertebral and non vertebral fractures.

\section{TIRES A PART}

\section{P.D. Delmas.}

